Rev. Inst. Flor. v. 27 n. 1 p. $83-101$ jun. 2015

http://dx.doi.org/10.4322/rif.2015.007

ISSN impresso 0103-2674/on-line 2178-5031

\title{
ORCHIDACEAE NO PARQUE ESTADUAL DA CANTAREIRA E SUA CONSERVAÇÃO'
}

\section{ORCHIDACEAE IN THE CANTAREIRA STATE PARK AND ITS CONSERVATION}

\author{
Luciano Ramos ZANDONÁ2,4; Eduardo Luís Martins CATHARINO³
}

\begin{abstract}
RESUMO - O Parque Estadual da Cantareira - PEC abriga uma das maiores florestas urbanas do mundo, declarada parte da Reserva da Biosfera do Cinturão Verde de São Paulo. A família Orchidaceae tem grande apelo ornamental e econômico, aspecto importante para a sensibilização de todos sobre a responsabilidade em conservar nossas espécies nativas e seus habitats. O presente trabalho visou, sobretudo, ao levantamento da família Orchidaceae no PEC. Para tal, foram realizadas coletas semanais, entre agosto de 2008 e fevereiro de 2014, utilizando o método da caminhada, tendo como base o mapa de fitofisionomias do PEC. A coleta de material botânico priorizou o resgate de plantas vivas em árvores e galhos caídos, sendo que as plantas foram incluídas em coleções vivas do Instituto de Botânica de São Paulo - IBt e PEC para acompanhamento da floração, herborização, documentação fotográfica e identificação. As plantas foram identificadas com base no material vivo utilizando-se da diagnose floral para determinação da espécie, as exsicatas foram tombadas no herbário do Instituto Florestal - SPSF. As demais plantas resgatadas, após identificação, foram realocadas no habitat e são também utilizadas em atividades educativas. Até o momento foram levantadas 159 espécies, de 64 gêneros, entre elas 10 espécies presentes em listas vermelhas, e outras dificilmente encontradas, o que coloca o PEC como detentor de maior riqueza de Orchidaceae da Região Metropolitana de São Paulo em comparação com outros levantamentos. Das 159 espécies registradas, 105 foram resgatadas, totalizando 3.120 indivíduos, dos quais 450 foram incluídos em coleções do IBt e PEC, e o restante realocado no habitat para observações e estudos futuros. Atividades com visitantes do PEC indicam que a utilização de orquídeas como tema para educação é bastante promissor.
\end{abstract}

Palavras-chave: biodiversidade; educação ambiental; florística; Mata Atlântica; São Paulo; Serra da Cantareira.

\begin{abstract}
The Cantareira State Park (Parque Estadual da Cantareira - PEC), declared part of the São Paulo Green Belt Biosphere Reserve, houses one of the largest urban forest in the world. The local Orchidaceae family has great ornamental and economic appeal, which is an important feature to raise the collective awareness and responsibility about the preservation of the native species and their habitat. The present study aimed, above all, to assess the Orchidaceae family within the PEC. Samples were taken weekley between August 2008 and February 2014 for this purpose using the walking method, based on the the PEC phytophysiognomies map. The collection of botanical material focused on the rescue of live plants that grow on trees and on fallen branches, and the plants were included subsequently in living collections of the Botanical Institute of São Paulo (IBt) and PEC for photographic documentation and identification as well as the monitoring of flowering and herborization.
\end{abstract}

\footnotetext{
1Trabalho originado da Dissertação de Mestrado em Biologia Vegetal, do primeiro autor, do Programa de Pós-Graduação em Biodiversidade Vegetal e Meio Ambiente do Instituto de Botânica de São Paulo. Recebido para análise em 20.10.14. Aceito para publicação em 04.05.15. Publicado on-line em 30.06.15.

²Orquidário Público de Guarulhos, Av. Papa João XXIII, 219, Parque Renato Maia, 07114-100 Guarulhos, SP, Brasil.

3Instituto de Botânica, Av. Miguel Stéfano, 3687, 04301-902 São Paulo, SP, Brasil.

${ }^{4}$ Autor para correspondência: Luciano Ramos Zandoná - luciano_zandona@yahoo.com.br
} 
The samples were identified based on living material, using the floral diagnosis to determine the species, and then exsiccated in the herbarium of the Forest Institute - SPSF. Other plants redeemed were relocated in the environment after identification and were also used in educational activities. One hundred fifty-nine species have been identified so far, 64 of them belong to different genera, 10 species are in red lists, and others are hardly ever found, which makes the Cantareira State Park the home to the greatest diversity of Orchidaceae in the São Paulo Metropolitan Region, compared to other surveys. Of the 159 species recorded, 105 were rescued, totaling 3,120 individuals. Four hundred fifty of them have been included both in the collections of the park as well as the Botanical Institute's, the remaining have been relocated in the environment for future studies and observations. The use of orchids as the theme for educational activities with the visitors to the Cantareira State Park proved to be very promising.

Keywords: biodiversity; environmental education; floristic; Atlantic Forest; São Paulo; Serra da Cantareira.

\section{INTRODUÇÃO}

A Mata Atlântica é considerada prioridade para a conservação da biodiversidade mundial e encontra-se entre os 34 "hotspots" do planeta (Myers et al., 2000). Seus remanescentes estão hoje reduzidos a pouco mais de $7 \%$ da cobertura original e, ainda assim, beneficiam milhares de pessoas com seus serviços ambientais como a amenização da temperatura, produção de água, proteção aos mananciais, fornecimento de polinizadores, barreira natural contra doenças e abrigo de rica biodiversidade (São Paulo, 2010).

A Mata Atlântica é um dos biomas com maior diversidade de epífitas das Américas, sendo a família Orchidaceae, na maioria das vezes, a mais numerosa entre as epífitas em ambientes úmidos tropicais, podendo também apresentar formas mico-heterotróficas, rupícolas e terrícolas (Hoehne, 1949; Catharino e Barros, 2004). A família é constituída por cerca de 800 gêneros e aproximadamente 25.000 espécies, na sua maioria (75\%) epífitas (Dressler, 1993; 2005). No Brasil, atualmente, são conhecidos mais de 200 gêneros e aproximadamente 2.500 espécies (Pabst e Dungs, 1975; 1977; Barros et al., 2014). Os levantamentos disponíveis e detalhados da família Orchidaceae, em São Paulo, são poucos, grande parte deles referindo-se às epífitas de um modo geral. Podem ser citados: Dislich e Mantovani (1998), que estudaram o componente epifítico vascular na mata da Reserva da Cidade Universitária "Armando de Salles Oliveira"; Piliackas et al. (2000), que estudaram as epífitas de manguezais paulistas; Breier (2005), que estudou as orquídeas do Parque Estadual Carlos Botelho, e Santos (2008), que realizou estudos de estrutura com epífitas no Parque Estadual das Fontes do Ipiranga - PEFI, listando poucas espécies de orquídeas; no entanto Barros (1983), em trabalho de florística, relacionou mais de cem espécies para a mesma área. Como levantamentos específicos para a família, além de Barros (1983), podemos citar: o levantamento das orquídeas do Maciço da Jureia e arredores realizado por Catharino e Barros (2004); Romanini e Barros (2008), que estudaram a família no Parque Estadual da Ilha do Cardoso, em Cananeia; Pansarin e Pansarin (2008), que realizaram um levantamento das Orchidaceae na Serra do Japi, Jundiaí (SP); Rodrigues e Barros (2012) estudaram as orquídeas no Parque Municipal Chiquinho Veríssimo, em Mogi das Cruzes.

A grande diversidade de espécies, formas e as diversas adaptações ecológicas da família, além de seu apelo ornamental, fazem das Orchidaceae um grupo potencial para conservação ex situ em jardins botânicos, sendo uma das famílias mais cultivadas em coleções de conservação ao redor do mundo (Stewart, 1991; Rassmussen e Rassmussen, 1991). As orquídeas, por serem adaptadas a nichos específicos e muito sensíveis a alterações do microclima, podem, de acordo com sua riqueza de espécies, indicar o grau de conservação de um ambiente e suas eventuais perturbações, prevenindo maiores danos à biodiversidade (Hietz, 1999).

Orchidaceae sofre grande pressão antrópica devido à coleta, comércio ilegal e supressão de habitats (Hoehne, 1949), estando entre as famílias de plantas mais coletadas ilegalmente em todo o mundo, apesar dos muitos esforços feitos atualmente para conservá-las, seja in situ ou ex situ, 
resgate, realocação, micropropagação, reintrodução de espécies e atividades de educação para conservação (Seaton, 2007; Mirenda, 2011). Estão listadas como ameaçadas para o Estado de São Paulo 128 espécies de Orchidaceae, em diversas categorias (São Paulo, 2004; Brasil, 2008; Martinelli e Moraes, 2013).

No $1^{\mathrm{o}}$ Congresso Internacional de Conservação de Orquídeas, realizado na Austrália, em 2001 (Mirenda, 2011), foram estabelecidas quatro diretrizes a serem seguidas, objetivando a conservação da família Orchidaceae em todo o mundo, são elas:

1. $90 \%$ das espécies ameaçadas em todo o mundo devem ser conservadas em coleções ex situ;

2. $50 \%$ das espécies ameaçadas devem estar incluídas em programas de conservação e recuperação, tendo como base o cultivo in vitro seguido de reintrodução destas espécies na natureza;

3. instruir e capacitar a comunidade local para o manejo sustentável, visando à propagação em grande escala e comércio de mudas cultivadas, com o intuito de diminuir a coleta ilegal de orquídeas nativas;

4. toda criança deve receber informações sobre a diversidade vegetal desde a alfabetização, inclusive sobre as orquídeas e sua importância para o equilíbrio ambiental.

Hoje, as orquídeas são referidas mundialmente como os "ursos panda" do reino vegetal, não havendo dúvida do seu potencial em despertar o interesse e o encanto das mais diversas pessoas, sendo assim, se torna possível utilizá-las como família ou espécie-bandeira e ferramenta educativa para a conservação das demais plantas e ambientes (Mirenda, 2011).

A Educação Ambiental é cada vez mais valorizada em todo mundo e, através da sua prática consciente, promove a preservação das características ambientais, necessárias à manutenção da vida de todos os seres (Honig, 2000). O processo de educação ambiental é lento e deve ser iniciado já na alfabetização das crianças (Dixon e Phyllips, 2007).
O Parque Estadual da Cantareira - PEC passou a integrar, em 1994, a Reserva do Cinturão Verde da Cidade de São Paulo, como Zona Núcleo, estando também inserido na Reserva da Biosfera da Mata Atlântica, ambas reconhecidas pelo programa "Man and Biosphere - MaB" da UNESCO. O Parque recebe em média 90.000 visitantes por ano (São Paulo, 2010), destes, $50 \%$ são estudantes e visitantes em atividades monitoradas por educadores ambientais, o que permite atribuir a tais visitas grande potencial para conscientização de estudantes e público em geral, moradores da Região Metropolitana de São Paulo, sobre a necessidade de conservação.

Por outro lado, não existem estudos específicos sobre a família Orchidaceae no PEC e a listagem disponível no Plano de Manejo da área relaciona apenas sete espécies de orquídeas (São Paulo, 2010), às quais podem ser acrescidos alguns registros no SpeciesLink. Desse modo, para que seja possível utilizar as orquídeas como ferramenta de educação para a conservação, auxiliando na sensibilização sobre a importância da preservação das florestas, da biodiversidade e dos recursos naturais renováveis, é necessário um aprofundamento no estudo da família na área.

Assim, o presente trabalho teve como objetivos: (1) efetuar o levantamento florístico da família Orchidaceae no PEC, consistindo de mapeamento, registro fotográfico e incorporação em herbário; (2) contribuir para a conservação de orquídeas do PEC apresentando informações sobre a abundância das espécies de orquídeas, sua representação em listas vermelhas e ocorrência nos diferentes núcleos/trilhas do PEC (destacando áreas de maior riqueza e com necessidade de conservação); (3) incrementar as coleções de conservação do Orquidário Frederico Carlos Hoehne e orquidário de visitação do PEC, com espécies raras e/ou ameaçadas da Região Metropolitana de São Paulo; (4) contribuir com propostas para utilização das orquídeas em ações de educação para conservação, apresentando ações de sensibilização e instrução de estudantes e visitantes do PEC quanto à necessidade de preservação das espécies e conservação dos recursos naturais renováveis. 


\section{MATERIAL E MÉTODOS}

\subsection{Localização e Caracterização da Área de Estudo}

O Parque Estadual da Cantareira, criado pelo Decreto Estadual $n^{\circ} 41.626$, de 30/11/1963, e pela Lei $\mathrm{n}^{\mathrm{o}} 10.228$, de 24/09/1968, abrange parte dos municípios de Caieiras, Guarulhos, Mairiporã e São Paulo, com a área de 7.916,52 ha.
Faz parte da Serra da Cantareira, cuja área totaliza aproximadamente 32.000 ha (Dantas, 1990) (Figura 1).

O Parque conta hoje com quatro núcleos: Águas Claras, Cabuçu, Engordador e Pedra Grande, todos eles tratados neste estudo (São Paulo, 2010). A região do Pinheirinho, embora não esteja aberta à visitação pública, também foi abordada neste estudo (Figura 2).

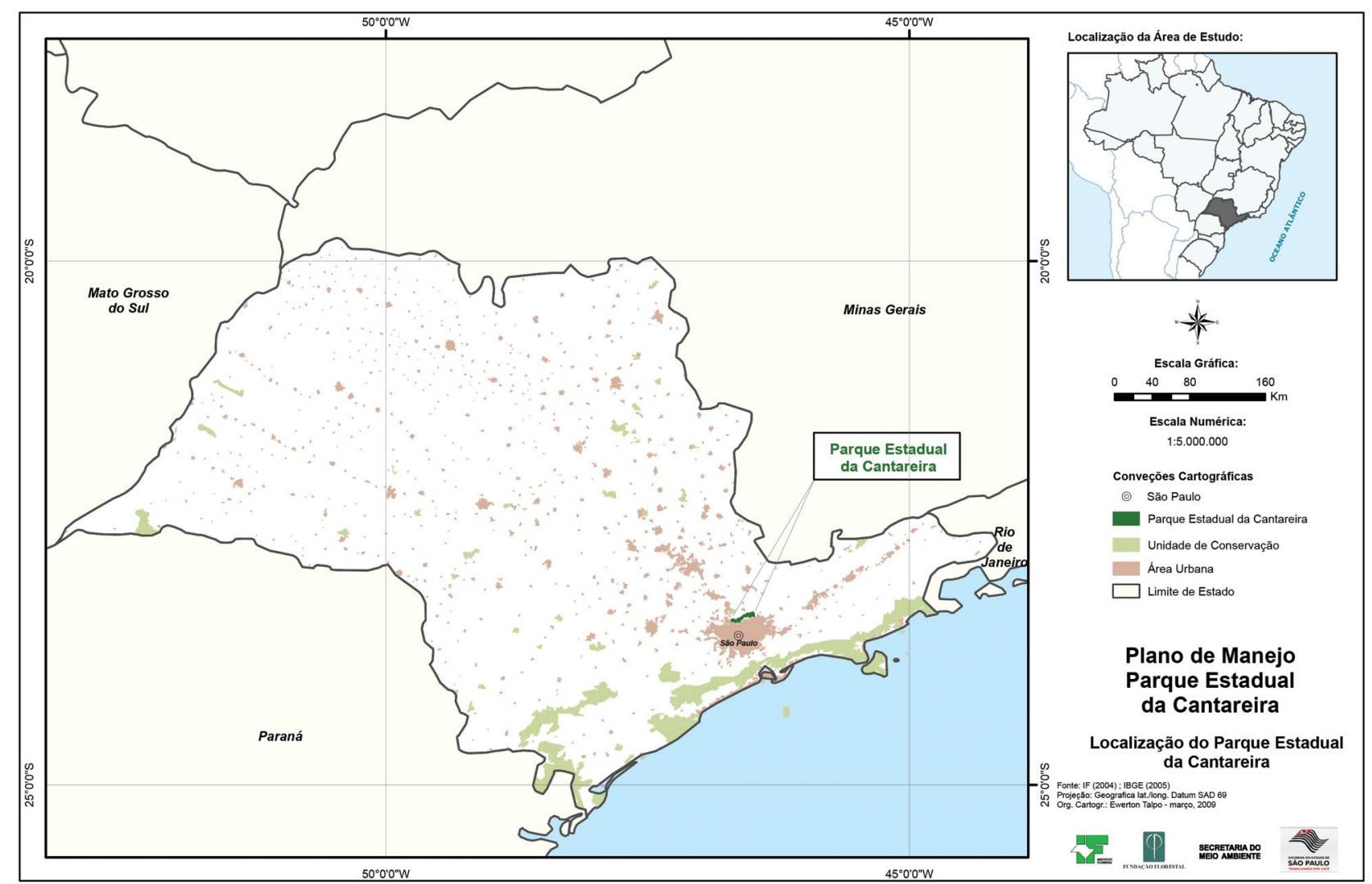

Figura 1. Localização do Parque Estadual da Cantareira, extraído do Plano de Manejo (São Paulo, 2010).

Figure 1. Location of the Cantareira State Park, extracted from the Management Plan (São Paulo, 2010). 


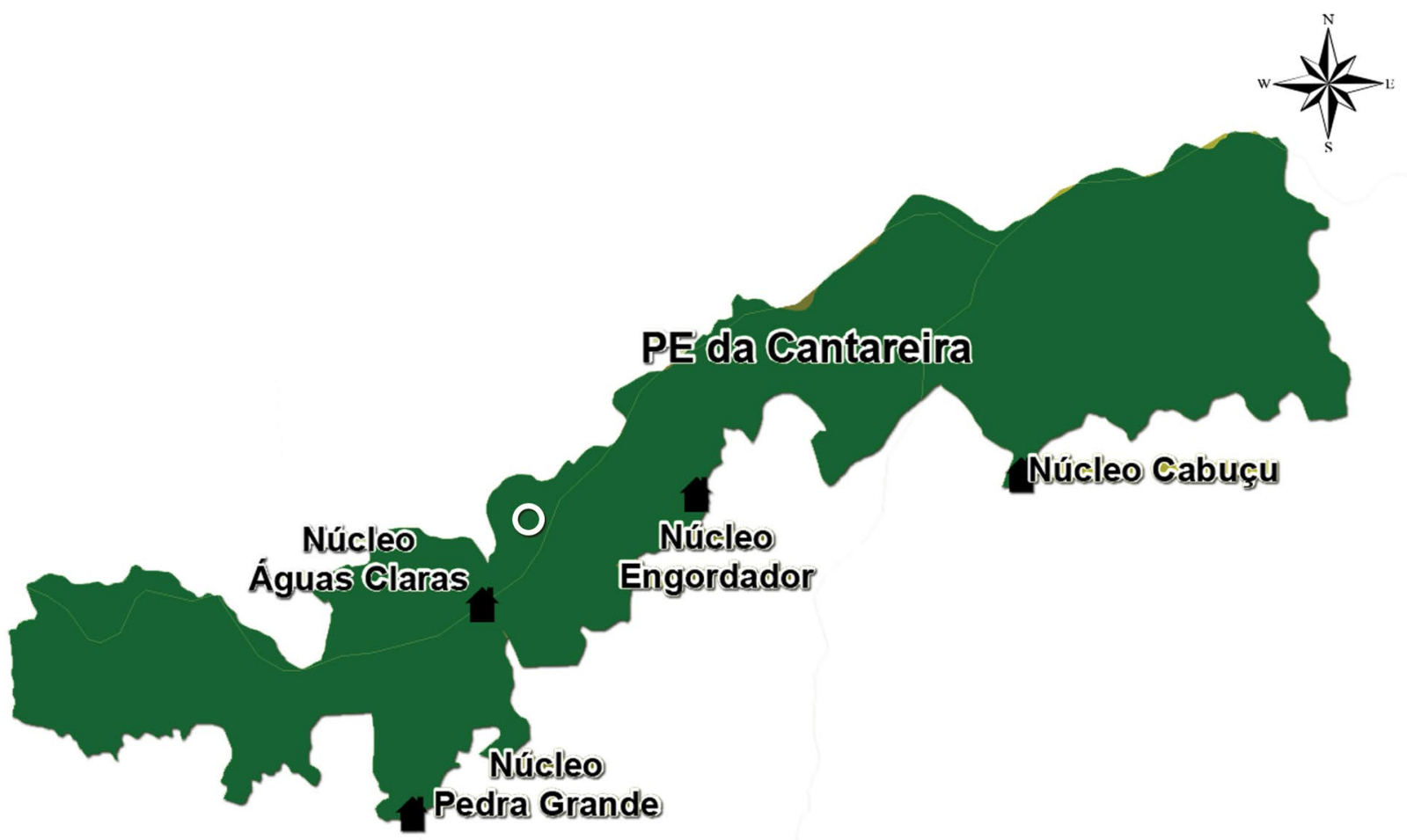

Figura 2. Localização dos núcleos do Parque Estadual da Cantareira e trilha do Pinheirinho indicada com círculo branco no mapa. Adaptado do Plano de Manejo (São Paulo, 2010).

Figure 2. Location of the Cantareira State Park nuclei and Pinheirinho track indicated by white circle on the map. Adapted from Management Plan (São Paulo, 2010).

O Parque Estadual da Cantareira - PEC é um dos principais remanescentes florestais de Mata Atlântica e abriga grande número de mananciais, sendo considerado uma das maiores florestas urbanas do planeta e protegido como Unidade de Conservação. O nome Cantareira vem de cântaro, vaso de barro, que era usado para armazenar água. Nessa região, com inúmeros córregos e nascentes, os cântaros eram depositados em prateleiras chamadas "cantareiras" (São Paulo, 2010). A proteção e conservação da Serra da Cantareira tiveram início no final do século XIX, época em que o governo do estado adquiriu algumas fazendas de café e chá, preocupado com o abastecimento de água da cidade de São Paulo que crescia rapidamente (São Paulo, 2010; Arzolla, 2011).

\subsection{Coletas, Herborização e Identificação}

Foram realizadas saídas de campo semanais, compreendendo o período de agosto de 2008 a fevereiro de 2014 em todos os núcleos do Parque Estadual da Cantareira - PEC: 
Águas Claras, Pedra Grande, Engordador e Cabuçú e na região do Pinheirinho, sendo priorizadas nos percursos as trilhas de uso público, trilhas de serviço, mas também foram realizadas caminhadas aleatórias em mata fechada tendo como base o mapa de fitofisionomias contido no Plano de Manejo do PEC (São Paulo, 2010).

O período de coletas botânicas foi de agosto de 2008 a fevereiro de 2014, as expedições a campo foram feitas quinzenalmente em percursos preestabelecidos ao longo das trilhas de visitação e também aleatoriamente, visando amostrar os trechos de cobertura vegetal mais preservada, tendo como base o mapa de fitofisionomias do plano de manejo do PEC, além de informações de especialistas do Instituto Florestal, utilizando a caminhada como método amostral (Filgueiras et al., 1994), compreendendo diversas situações de relevo, declividade e altitude.

A obtenção de material botânico foi feita preferencialmente com base no resgate de plantas em árvores e galhos caídos. As espécies encontradas, mas não obtidas durante as atividades de resgate, foram observadas em seu habitat, quando necessário por meio da escalada, sendo fotografadas quando floridas, e tendo uma frente fértil coletada para herborização, seguindo-se as técnicas convencionais, e posterior depósito no Herbário do Instituto Florestal - SPSF. Apesar de as plantas avistadas no habitat terem sido georreferenciadas, dados exatos de localização não serão apresentados, procurando-se, com isto, evitar coletas indevidas, tendo em vista que orquídeas são comumente visadas por colecionadores e muitas espécies ocorrem em número reduzido de indivíduos. Além das coletas foi realizada consulta à rede SpeciesLink (SpeciesLink, 2014), para complementar as informações sobre ocorrência para a área de estudo.

\subsection{Organização e Análise dos Dados}

Ao fim do mapeamento e documentação fotográfica, foi elaborada uma listagem geral das espécies coletadas e/ou observadas, organizada de acordo com Pabst e Dungs (1975; 1977), para todos os grupos, contendo informações sobre as coletas, documentação fotográfica, quantificação preliminar da ocorrência das espécies nas diferentes áreas do Parque, entre outras, sendo as espécies classificadas em epífitas, terrícolas ou rupícolas. Adotou-se Pabst e Dungs $(1975 ; 1977)$ tendo em vista as grandes mudanças nomenclaturais ocorridas nos últimos anos. Em Zandoná (2014), encontra-se uma comparação da listagem de Pabst e Dungs $(1975$; 1977) e da lista das espécies da Flora do Brasil (Barros et al., 2014).

As espécies foram classificadas segundo seu status ou grau de ameaça, de acordo com as listas oficiais de espécies em extinção para São Paulo e para o Brasil (São Paulo, 2004; Brasil, 2008; Martinelli e Moraes; 2013). Foram utilizadas as seguintes categorias de ameaça: Presumivelmente Extinta (EX), Presumivelmente Extinta na Natureza (EW), Em Perigo Crítico (CR), Em Perigo (EN), Vulnerável (VU) e Pouco Preocupante (LC).

Para avaliar a abundância de indivíduos de cada espécie no habitat, foi adotado o que se denominou "categorias de abundância", definindo classes de acordo com a riqueza de espécies avistadas e resgatadas, visualização de frutos e presença de plântulas nas proximidades de vegetais adultos no habitat nas diferentes áreas. Foram definidas três categorias: abundante, ocasional e rara. Foram consideradas dentro da categoria "abundante" aquelas plantas encontradas em todos os núcleos, mesmo em áreas sujeitas à ação antrópica, resgatadas ou visualizadas no habitat em número igual ou superior a 50 indivíduos, frutos sempre presentes, plântulas se desenvolvendo sobre as raízes e proximidades. $\mathrm{Na}$ categoria "ocasional", foram incluídas as plantas não encontradas em todos os núcleos, somente registradas em áreas de florestas em estágio de regeneração avançado ou maduro, resgatadas ou visualizadas no habitat entre 11 e 49 indivíduos, frutos dificilmente visualizados, poucas ou nenhuma plântula aderida às raízes e proximidades. $\mathrm{Na}$ categoria "rara", foram incluídas as plantas não encontradas em todos os núcleos, somente registradas em áreas de floresta madura, resgatadas ou visualizadas no habitat em número igual ou inferior a dez indivíduos, frutos não visualizados, nenhuma plântula sobre as raízes ou proximidades. 


\subsection{Análises de Similaridade}

A similaridade da flora amostrada entre os núcleos do PEC foi avaliada pela presença das espécies em comum entre eles através de análises multivariadas de agrupamento, utilizando basicamente o método UPGMA (Unweighted Pair Group Method with Arithmetic Mean) com o coeficiente de Jaccard, através da utilização do aplicativo PAST (Hammer et al., 2001).

\subsection{Conservação in situ: realocação de plantas na natureza}

Plantas grandes resgatadas foram automaticamente realocadas em árvores próximas ao local onde foram encontradas, utilizando para tal fim a ascensão por corda, quando necessário. As micro-orquídeas, mais sensíveis e exigentes quanto à umidade, foram realocadas em árvores próximas, que ofereciam as condições apropriadas a cada espécie, para observação futura, obtenção de fotografias e material a ser herborizado. A fixação das plantas foi feita utilizando-se de fitilho ou barbante encerado, que foram removidos assim que a planta se fixou pelas próprias raízes.

\subsection{Conservação ex situ: inclusão de plantas em coleções vivas}

As plantas provenientes do resgate foram mantidas em cultivo nas coleções vivas do PEC e no Orquidário Frederico Carlos Hoehne - $\mathrm{OFCH}$, fiel depositário do patrimônio genético pelo CENARGEN, para conservação, identificação e herborização, bem como foram levantadas as plantas já existentes coletadas na Região Metropolitana de São Paulo nas coleções vivas, herbários e bibliografia. Essas plantas tiveram acompanhamento da floração para fotografias e posterior herborização da frente florida. Foi sempre priorizada a conservação da planta viva, sendo que para as plantas pouco desenvolvidas foi coletada somente a inflorescência.
Tendo em vista o estado de muitas das plantas resgatadas, adotou-se como metodologia complementar um tratamento fitossanitário padrão, imergindo as plantas em solução de hipoclorito de sódio a $1 \%$ por 10 minutos para desinfecção e aplicação de canela em pó sobre o rizoma, por suas propriedades: bactericida, fungicida, cicatrizante e estimuladora de enraizamento e brotação. Depois desse tratamento, as plantas permaneceram sobre bancada seca por uma semana, sendo posteriormente levadas para cultivo.

\subsection{Atividades de Educação para a Conservação}

Foram propostas e elaboradas atividades que incluíssem as orquídeas no roteiro de visitação do PEC, entre elas, a utilização da coleção viva, utilização de observadores feitos de bambu adaptados de (Honig, 2000), realocação de plantas resgatadas durante as visitas monitoradas, caminhadas para observação de plantas no habitat e elaboração de material didático de apoio.

Os educadores do PEC foram capacitados a utilizar as orquídeas como ferramenta educativa em visitas monitoradas na coleção viva e nas trilhas guiadas, para isto, foram treinados para a observação e identificação das orquídeas avistadas nas trilhas e coleção viva do PEC para despertar a curiosidade dos visitantes, atividades fundamentais ao aprendizado sobre a necessidade de se conhecer e conservar plantas em seu habitat.

\section{RESULTADOS E DISCUSSÃO}

Registraram-se 150 espécies ou morfoespécies de Orchidaceae na área estudada. A rede SpeciesLink (SpeciesLink, 2014) registra 37 ocorrências para a área que, após revistas as sinonimizações e retiradas as ocorrências repetidas, adicionou mais nove espécies à listagem, totalizando 159 espécies distribuídas em 64 gêneros, destas, 121 são epífitas e 38 terrícolas. A listagem das espécies registradas, incluindo o hábito e as categorias de ocorrência, encontra-se na Tabela 1. 
Tabela 1. Orchidaceae registradas no Parque Estadual da Cantareira, São Paulo, Brasil, núcleos: Águas Claras - AC, Cabuçú - C, Engordador - E, Pedra Grande - PG, Pinheirinho - P e registros no SpeciesLink - SL (identificações baseadas em Pabst e Dungs, 1975; 1977). Espécies em listas vermelhas em negrito; hábito de vida - Hb: epífita - E, terrícola $-\mathrm{T}$, rupícola $-\mathrm{R}$; categorias de ocorrência - Oc: abundante $-\mathrm{A}$, ocasional - $\mathrm{O}$ e rara $-\mathrm{R}$.

Table 1. Orchidaceae recorded in the Cantareira State Park, São Paulo, Brazil, nuclei: Águas Claras - AC, Cabuçu - C, Engordador - E, Pedra Grande - PG, Pinheirinho - P and records in SpeciesLink - SL (names based on Pabst and Dungs, 1975; 1977). Species in red lists in bold; life habit - Hb: epiphytic - E, terrestrial - T, rupicolous - R; categories of occurrence - Oc: abundant $-\mathrm{A}$, occasional $-\mathrm{O}$ and rare $-\mathrm{R}$.

\begin{tabular}{|c|c|c|c|c|c|c|c|c|c|}
\hline $\mathrm{N}$ & Espécie & $\mathrm{Hb}$ & Oc & $\mathrm{C}$ & $\mathrm{E}$ & $\mathrm{PG}$ & $\mathrm{P}$ & $\mathrm{AC}$ & SL \\
\hline 1 & Amblostoma armeniacum (Lindl.) Brieger & $\mathrm{E}$ & $\mathrm{R}$ & $\mathrm{x}$ & $\mathrm{x}$ & $\mathrm{x}$ & $\mathrm{x}$ & & $\mathrm{x}$ \\
\hline 2 & Amblostoma tridactylum (Lindl.) Rchb.f. & $\mathrm{E}$ & $\mathrm{R}$ & & & $\mathrm{x}$ & & & \\
\hline 3 & Barbosella australis (Cogn.) Schltr. & $\mathrm{E}$ & $\mathrm{R}$ & $\mathrm{x}$ & $\mathrm{x}$ & $\mathrm{x}$ & $\mathrm{x}$ & & \\
\hline 4 & Barbosella dusenii (A.Samp.) Schltr. & $\mathrm{E}$ & $\mathrm{R}$ & $\mathrm{x}$ & $\mathrm{x}$ & $\mathrm{x}$ & $\mathrm{x}$ & & \\
\hline 5 & Barbosella microphylla (Barb.Rodr.) Schltr. & $\mathrm{E}$ & $\mathrm{O}$ & $\mathrm{x}$ & $\mathrm{x}$ & & $\mathrm{x}$ & & \\
\hline 6 & Barbosella miersii (Lindl.) Schltr. & $\mathrm{E}$ & $\mathrm{R}$ & & $\mathrm{x}$ & & $\mathrm{x}$ & & \\
\hline 7 & Bifrenaria aureofulva Lindl. & $\mathrm{E}$ & $\mathrm{O}$ & $\mathrm{x}$ & $\mathrm{x}$ & $\mathrm{x}$ & $\mathrm{x}$ & $\mathrm{x}$ & \\
\hline 8 & Bifrenaria harrisoniae (Hook.) Rchb.f. & $\mathrm{E}$ & $\mathrm{O}$ & $\mathrm{x}$ & $\mathrm{x}$ & $\mathrm{x}$ & $\mathrm{x}$ & $\mathrm{x}$ & \\
\hline 9 & Bulbophyllum napellii Lindl. & $\mathrm{E}$ & $\mathrm{R}$ & & & & $\mathrm{x}$ & & \\
\hline 10 & Bulbophyllum sp. & $\mathrm{E}$ & $\mathrm{R}$ & & & $\mathrm{x}$ & & & \\
\hline 11 & Campylocentrum aromaticum Barb.Rodr. & $\mathrm{E}$ & $\mathrm{O}$ & $\mathrm{x}$ & $\mathrm{x}$ & $\mathrm{x}$ & & $\mathrm{x}$ & \\
\hline 12 & Campylocentrum crassirhizum Hoehne & $\mathrm{E}$ & $\mathrm{R}$ & $\mathrm{x}$ & $\mathrm{x}$ & & & $\mathrm{x}$ & \\
\hline 13 & Campylocentrum pauloense Hoehne e Schltr. & $\mathrm{E}$ & $\mathrm{R}$ & $\mathrm{x}$ & & & & & $\mathrm{x}$ \\
\hline 14 & Campylocentrum sp.1 & $\mathrm{E}$ & $\mathrm{R}$ & $\mathrm{x}$ & & $\mathrm{x}$ & $\mathrm{x}$ & & \\
\hline 15 & Capanemia gehrtii Hoehne & $\mathrm{E}$ & $\mathrm{R}$ & $\mathrm{x}$ & $\mathrm{x}$ & $\mathrm{x}$ & $\mathrm{x}$ & $\mathrm{x}$ & \\
\hline 16 & Capanemia micromera Barb.Rodr. & $\mathrm{E}$ & $\mathrm{R}$ & $\mathrm{x}$ & $\mathrm{x}$ & $\mathrm{x}$ & $\mathrm{x}$ & $\mathrm{x}$ & \\
\hline 17 & Capanemia superflua (Rchb.f.) Garay & $\mathrm{E}$ & $\mathrm{O}$ & & & $\mathrm{x}$ & & & \\
\hline 18 & Capanemia theresae Barb.Rodr. & $\mathrm{E}$ & $\mathrm{O}$ & $\mathrm{x}$ & $\mathrm{x}$ & $\mathrm{x}$ & $\mathrm{x}$ & $\mathrm{x}$ & \\
\hline 19 & Catasetum cernuum (Lindl.) Rchb.f. & $\mathrm{E}$ & $\mathrm{O}$ & $\mathrm{x}$ & $\mathrm{x}$ & $\mathrm{x}$ & & & \\
\hline 20 & Catasetum trulla Lindl. & $\mathrm{E}$ & $\mathrm{R}$ & & & $\mathrm{x}$ & & & \\
\hline 21 & Centroglossa macroceras Barb.Rodr. & $\mathrm{E}$ & $\mathrm{R}$ & $\mathrm{x}$ & $\mathrm{x}$ & $\mathrm{x}$ & $\mathrm{x}$ & $\mathrm{x}$ & $\mathrm{x}$ \\
\hline 22 & Cleistes gracilis Schltr. & $\mathrm{T}$ & & & & & & & $\mathrm{x}$ \\
\hline 23 & Comparettia coccinea Lindl. & $\mathrm{E}$ & $\mathrm{R}$ & $\mathrm{x}$ & $\mathrm{x}$ & $\mathrm{x}$ & $\mathrm{x}$ & & \\
\hline 24 & Corymborkis flava (Sw.) Kuntze & $\mathrm{T}$ & $\mathrm{R}$ & $\mathrm{x}$ & $\mathrm{x}$ & & $\mathrm{x}$ & & $\mathrm{x}$ \\
\hline 25 & Cranichis candida (Barb.Rodr.) Cogn. & $\mathrm{T}$ & $\mathrm{R}$ & $\mathrm{x}$ & & & $\mathrm{x}$ & & \\
\hline 26 & Cyclopogon bicolor (Ker-Gawl.) Schltr. & $\mathrm{T}$ & $\mathrm{R}$ & & $\mathrm{x}$ & $\mathrm{x}$ & & $\mathrm{x}$ & \\
\hline 27 & Cyclopogon congestus (Vell.) Hoehne & $\mathrm{T}$ & A & & & $\mathrm{x}$ & & $\mathrm{x}$ & \\
\hline 28 & Cyclopogon elegans Hoehne & $\mathrm{T}$ & $\mathrm{R}$ & & $\mathrm{x}$ & $\mathrm{x}$ & & $\mathrm{x}$ & \\
\hline 29 & Cyclopogon iguapensis Schltr. & $\mathrm{T}$ & & & & & & & $\mathrm{x}$ \\
\hline 30 & Cyclopogon longibracteatus (Barb.Rodr.) Schltr. & $\mathrm{T}$ & $\mathrm{R}$ & $\mathrm{x}$ & $\mathrm{x}$ & $\mathrm{x}$ & $\mathrm{x}$ & $\mathrm{x}$ & \\
\hline
\end{tabular}

continua to be continued 
continuação - Tabela 1

continuation - Table 1

\begin{tabular}{|c|c|c|c|c|c|c|c|c|c|}
\hline $\mathrm{N}$ & Espécie & $\mathrm{Hb}$ & Oc & $\mathrm{C}$ & $\mathrm{E}$ & PG & $\mathrm{P}$ & $\mathrm{AC}$ & SL \\
\hline 31 & Cyclopogon variegatus Barb.Rodr. & $\mathrm{T}$ & $\mathrm{O}$ & $\mathrm{x}$ & $\mathrm{x}$ & & & & \\
\hline 32 & Cyclopogon warmingii (Rchb.f.) Schltr. & $\mathrm{T}$ & & & & & & & $\mathrm{x}$ \\
\hline 33 & Cyglopogon sp. & $\mathrm{T}$ & $\mathrm{R}$ & $\mathrm{x}$ & & & & & \\
\hline 34 & Dichaea cogniauxiana Schltr. & $\mathrm{E}$ & $\mathrm{O}$ & $\mathrm{x}$ & $\mathrm{x}$ & $\mathrm{x}$ & $\mathrm{x}$ & & \\
\hline 35 & Dichaea pendula (Aubl.) Cogn. & $\mathrm{E}$ & $\mathrm{R}$ & $\mathrm{x}$ & & & & & \\
\hline 36 & Eltroplectris cf. calcarata (Sw.) Garay e Sweet & $\mathrm{T}$ & $\mathrm{R}$ & $\mathrm{x}$ & & & & & \\
\hline 37 & Encyclia inversa (Lindl.) Pabst & $\mathrm{E}$ & $\mathrm{R}$ & & $\mathrm{x}$ & & $\mathrm{x}$ & & $\mathrm{x}$ \\
\hline 38 & Encyclia patens Hook. & $\mathrm{E}$ & $\mathrm{O}$ & $\mathrm{x}$ & $\mathrm{x}$ & $\mathrm{x}$ & $\mathrm{x}$ & $\mathrm{x}$ & \\
\hline 39 & Epidendrum campaccii Hágsater e L.Sánchez & $\mathrm{E}$ & $\mathrm{O}$ & $\mathrm{x}$ & $\mathrm{x}$ & $\mathrm{x}$ & $\mathrm{x}$ & $\mathrm{x}$ & $\mathrm{x}$ \\
\hline 40 & Epidendrum densiflorum Hook. & & $\mathrm{R}$ & & & & & & $\mathrm{x}$ \\
\hline 41 & Epidendrum proligerum Barb.Rodr. & $\mathrm{E}$ & $\mathrm{R}$ & & & $\mathrm{x}$ & $\mathrm{x}$ & & \\
\hline 42 & Epidendrum ramosum Jacq. & $\mathrm{E}$ & $\mathrm{R}$ & & & $\mathrm{x}$ & & & \\
\hline 43 & Epidendrum rigidum Jacq. & $\mathrm{E}$ & $\mathrm{R}$ & $\mathrm{x}$ & $\mathrm{x}$ & $\mathrm{x}$ & $\mathrm{x}$ & $\mathrm{x}$ & \\
\hline 44 & Epidendrum secundum Jacq. & $\mathrm{E} / \mathrm{R}$ & A & $\mathrm{x}$ & $\mathrm{x}$ & $\mathrm{x}$ & $\mathrm{x}$ & $\mathrm{x}$ & $\mathrm{x}$ \\
\hline 45 & Erythrodes lacteola (Barb.Rodr.) Ames & $\mathrm{T}$ & $\mathrm{R}$ & $\mathrm{x}$ & $\mathrm{x}$ & & $\mathrm{x}$ & & \\
\hline 46 & Erythrodes picta (Lindl.) Ames & $\mathrm{T}$ & $\mathrm{R}$ & $\mathrm{x}$ & & & & & \\
\hline 47 & Erythrodes rosea (Lindl.) & $\mathrm{T}$ & $\mathrm{R}$ & & & $\mathrm{x}$ & $\mathrm{x}$ & & \\
\hline 48 & Eulophia alta (L.) Fawc. e Rendle & $\mathrm{T}$ & $\mathrm{O}$ & $\mathrm{x}$ & $\mathrm{x}$ & $\mathrm{x}$ & $\mathrm{x}$ & $\mathrm{x}$ & \\
\hline 49 & Eurystyles actinosophila (Barb.Rodr.) Schltr. & $\mathrm{E}$ & $\mathrm{O}$ & $\mathrm{x}$ & $\mathrm{x}$ & $\mathrm{x}$ & $\mathrm{x}$ & $\mathrm{x}$ & \\
\hline 50 & Eurystyles cotyledon Wawra & $\mathrm{E}$ & $\mathrm{R}$ & $\mathrm{x}$ & $\mathrm{x}$ & $\mathrm{x}$ & $\mathrm{x}$ & $\mathrm{x}$ & $\mathrm{x}$ \\
\hline 51 & Eurystyles sp. & $\mathrm{E}$ & $\mathrm{R}$ & & & $\mathrm{x}$ & & & \\
\hline 52 & Galeandra beyrichii Rchb. f. & $\mathrm{T}$ & $\mathrm{R}$ & & & $\mathrm{x}$ & & & \\
\hline 53 & Gomesa crispa (Lindl.) Klotzsch ex Rchb.f. & $\mathrm{E}$ & A & $\mathrm{x}$ & $\mathrm{x}$ & $\mathrm{x}$ & $\mathrm{x}$ & $\mathrm{x}$ & $\mathrm{x}$ \\
\hline 54 & Gomesa laxiflora (Lindl.) Klotzsch ex Rchb.f. & $\mathrm{E}$ & & & & & & & $\mathrm{x}$ \\
\hline 55 & Gomesa recurva Lodd. & $\mathrm{E}$ & A & $\mathrm{x}$ & $\mathrm{x}$ & $\mathrm{x}$ & $\mathrm{x}$ & $\mathrm{x}$ & $\mathrm{x}$ \\
\hline 56 & Govenia utriculata (Sw.) Lindl. & $\mathrm{T}$ & $\mathrm{R}$ & & & $\mathrm{x}$ & & & $\mathrm{x}$ \\
\hline 57 & Grobya amherstiae Lindl. & $\mathrm{E}$ & A & $\mathrm{x}$ & $\mathrm{x}$ & $\mathrm{x}$ & $\mathrm{x}$ & $\mathrm{x}$ & \\
\hline 58 & Habenaria cf. paranaensis Barb.Rodr. & $\mathrm{T}$ & $\mathrm{R}$ & & & $\mathrm{x}$ & & & $\mathrm{x}$ \\
\hline 59 & Habenaria fastor Lindl. ex Warm. & $\mathrm{T}$ & $\mathrm{R}$ & & $\mathrm{x}$ & & & & \\
\hline 60 & Habenaria josephensis Barb.Rodr. & $\mathrm{T}$ & $\mathrm{O}$ & $\mathrm{x}$ & $\mathrm{x}$ & $\mathrm{x}$ & $\mathrm{x}$ & $\mathrm{x}$ & \\
\hline 61 & Habenaria parviflora Lindl. & $\mathrm{T}$ & $\mathrm{O}$ & & & $\mathrm{x}$ & $\mathrm{x}$ & & \\
\hline 62 & Habenaria pleiophylla Hoehne e Schltr. & $\mathrm{T}$ & $\mathrm{O}$ & & $\mathrm{x}$ & $\mathrm{x}$ & $\mathrm{x}$ & & $\mathrm{x}$ \\
\hline 63 & Habenaria repens Nutt. & $\mathrm{T}$ & & & & & & & $\mathrm{x}$ \\
\hline 64 & Habenaria sp.1 & $\mathrm{T}$ & $\mathrm{R}$ & & & & $\mathrm{x}$ & & \\
\hline 65 & Hapalorchis lineatus (Lindl.) Schltr. & $\mathrm{T}$ & $\mathrm{R}$ & & & $\mathrm{x}$ & & $\mathrm{x}$ & \\
\hline 66 & Isabelia virginalis Barb.Rodr. & $\mathrm{E}$ & $\mathrm{R}$ & & & $\mathrm{x}$ & & & \\
\hline 67 & Isochilus linearis (Jacq.) R.Br. & $\mathrm{E}$ & $\mathrm{O}$ & $\mathrm{x}$ & $\mathrm{x}$ & $\mathrm{x}$ & $\mathrm{x}$ & $\mathrm{x}$ & \\
\hline 68 & Laelia virens Lindl. & $\mathrm{E}$ & $\mathrm{R}$ & & & $\mathrm{x}$ & & & $\mathrm{x}$ \\
\hline
\end{tabular}


continuação - Tabela 1

continuation - Table 1

\begin{tabular}{|c|c|c|c|c|c|c|c|c|c|}
\hline $\mathrm{N}$ & Espécie & $\mathrm{Hb}$ & Oc & $\mathrm{C}$ & $\mathrm{E}$ & PG & $\mathrm{P}$ & $\mathrm{AC}$ & SL \\
\hline 69 & Lankesterella epiphyta (Barb.Rodr.) Mansf. & $\mathrm{E}$ & $\mathrm{R}$ & & & $\mathrm{x}$ & & & \\
\hline 70 & Leptotes bicolor Lindl. & $\mathrm{E}$ & $\mathrm{O}$ & $\mathrm{x}$ & $\mathrm{x}$ & $\mathrm{x}$ & $\mathrm{x}$ & $\mathrm{x}$ & \\
\hline 71 & Liparis nervosa (Thunb.) Lindl. & $\mathrm{T}$ & $\mathrm{O}$ & $\mathrm{x}$ & $\mathrm{x}$ & $\mathrm{x}$ & $\mathrm{x}$ & $\mathrm{x}$ & \\
\hline 72 & Lycaste rossyi Hoehne & $\mathrm{E}$ & $\mathrm{R}$ & & $\mathrm{x}$ & & & & \\
\hline 73 & Malaxis excavata (Lindl.) Kuntze & $\mathrm{T}$ & $\mathrm{R}$ & $\mathrm{x}$ & & $\mathrm{x}$ & $\mathrm{x}$ & $\mathrm{x}$ & \\
\hline 74 & Malaxis parthoni C.Morren & $\mathrm{T}$ & $\mathrm{R}$ & & & & & & $\mathrm{x}$ \\
\hline 75 & Maxillaria cerifera Barb.Rodr. & $\mathrm{E}$ & $\mathrm{O}$ & $\mathrm{x}$ & & & $\mathrm{x}$ & & \\
\hline 76 & Maxillaria cogniauxiana Hoehne & $\mathrm{E}$ & $\mathrm{O}$ & & $\mathrm{x}$ & $\mathrm{x}$ & $\mathrm{x}$ & & \\
\hline 77 & Maxillaria gracilis Lodd. & $\mathrm{E}$ & $\mathrm{O}$ & $\mathrm{x}$ & $\mathrm{x}$ & $\mathrm{x}$ & $\mathrm{x}$ & & \\
\hline 78 & Maxillaria madida Lindl. & $\mathrm{E}$ & $\mathrm{O}$ & & & $\mathrm{x}$ & $\mathrm{x}$ & & \\
\hline 79 & Maxillaria marginata (Lindl.) Fenzl & $\mathrm{E}$ & $\mathrm{R}$ & $\mathrm{x}$ & & & & & \\
\hline 80 & Maxillaria picta Hook. & $\mathrm{E}$ & $\mathrm{O}$ & & $\mathrm{x}$ & $\mathrm{x}$ & $\mathrm{x}$ & & \\
\hline 81 & Mesadenella cuspidata (Lindl.) Garay & $\mathrm{T}$ & $\mathrm{R}$ & $\mathrm{x}$ & $\mathrm{x}$ & $\mathrm{x}$ & $\mathrm{x}$ & $\mathrm{x}$ & \\
\hline 82 & Miltonia regnellii Rchb.f. & $\mathrm{E}$ & & & & & $\mathrm{x}$ & & $\mathrm{x}$ \\
\hline 83 & Notylia longispicata Hoehne e Schltr. & $\mathrm{E}$ & $\mathrm{O}$ & $\mathrm{x}$ & $\mathrm{x}$ & $\mathrm{x}$ & $\mathrm{x}$ & $\mathrm{x}$ & $\mathrm{x}$ \\
\hline 84 & Octomeria cf. palmyrabellae Barb.Rodr. & $\mathrm{E}$ & $\mathrm{R}$ & & & $\mathrm{x}$ & & & \\
\hline 85 & Octomeria concolor Barb.Rodr. & $\mathrm{E} / \mathrm{R}$ & $\mathrm{R}$ & & & $\mathrm{x}$ & & & \\
\hline 86 & Octomeria crassifolia Lindl. & $\mathrm{E}$ & $\mathrm{O}$ & $\mathrm{x}$ & $\mathrm{x}$ & $\mathrm{x}$ & $\mathrm{x}$ & $\mathrm{x}$ & \\
\hline 87 & Octomeria diaphana Lindl. & $\mathrm{E}$ & $\mathrm{R}$ & & $\mathrm{x}$ & & $\mathrm{x}$ & & \\
\hline 88 & Octomeria gracilis Lodd. ex Lindl. & $\mathrm{E}$ & $\mathrm{R}$ & & & $\mathrm{x}$ & & & \\
\hline 89 & Octomeria grandiflora Lindl. & $\mathrm{E}$ & $\mathrm{O}$ & $\mathrm{x}$ & $\mathrm{x}$ & $\mathrm{x}$ & $\mathrm{x}$ & & $\mathrm{x}$ \\
\hline 90 & Octomeria juncifolia Barb.Rodr. & $\mathrm{E}$ & $\mathrm{R}$ & & & & $\mathrm{x}$ & & \\
\hline 91 & Oeceoclades maculata (Lindl.) Lindl. & $\mathrm{T}$ & A & $\mathrm{x}$ & $\mathrm{x}$ & $\mathrm{x}$ & $\mathrm{x}$ & $\mathrm{x}$ & \\
\hline 92 & Oncidium crispum Lodd. & $\mathrm{E}$ & $\mathrm{R}$ & $\mathrm{x}$ & $\mathrm{x}$ & $\mathrm{x}$ & & & \\
\hline 93 & Oncidium divaricatum (Lindl.) Beer & $\mathrm{E}$ & $\mathrm{R}$ & & & $\mathrm{x}$ & & & \\
\hline 94 & Oncidium flexuosum (Kunth) Lindl. & $\mathrm{E}$ & A & $\mathrm{x}$ & $\mathrm{x}$ & $\mathrm{x}$ & $\mathrm{x}$ & $\mathrm{x}$ & \\
\hline 95 & Oncidium forbesii Hook. & $\mathrm{E}$ & $\mathrm{R}$ & $\mathrm{x}$ & & $\mathrm{x}$ & $\mathrm{x}$ & & \\
\hline 96 & Oncidium gardneri Lindl. & $\mathrm{E}$ & $\mathrm{R}$ & & & $\mathrm{x}$ & $\mathrm{x}$ & & \\
\hline 97 & Oncidium harrisonianum Lindl. & $\mathrm{E}$ & $\mathrm{R}$ & & & $\mathrm{x}$ & & & \\
\hline 98 & Oncidium hians Lindl. & $\mathrm{E}$ & $\mathrm{R}$ & & & $\mathrm{x}$ & & & \\
\hline 99 & Oncidium hookeri Rolfe & $\mathrm{E}$ & $\mathrm{O}$ & $\mathrm{x}$ & $\mathrm{x}$ & $\mathrm{x}$ & $\mathrm{x}$ & $\mathrm{x}$ & $\mathrm{x}$ \\
\hline 100 & Oncidium longipes Lindl. & $\mathrm{E}$ & $\mathrm{R}$ & $\mathrm{x}$ & $\mathrm{x}$ & $\mathrm{x}$ & $\mathrm{x}$ & $\mathrm{x}$ & \\
\hline 101 & Oncidium praetextum Rchb.f. & $\mathrm{E}$ & $\mathrm{R}$ & $\mathrm{x}$ & $\mathrm{x}$ & $\mathrm{x}$ & $\mathrm{x}$ & & \\
\hline 102 & Oncidium pubes Lindl. & $\mathrm{E}$ & $\mathrm{O}$ & $\mathrm{x}$ & $\mathrm{x}$ & $\mathrm{x}$ & $\mathrm{x}$ & $\mathrm{x}$ & \\
\hline 103 & Oncidium pumilum Lindl. & $\mathrm{E}$ & A & $\mathrm{x}$ & $\mathrm{x}$ & $\mathrm{x}$ & $\mathrm{x}$ & $\mathrm{x}$ & \\
\hline
\end{tabular}


continuação - Tabela 1

continuation - Table 1

\begin{tabular}{|c|c|c|c|c|c|c|c|c|c|}
\hline $\mathrm{N}$ & Espécie & $\mathrm{Hb}$ & Oc & $\mathrm{C}$ & $\mathrm{E}$ & PG & $\mathrm{P}$ & $\mathrm{AC}$ & SL \\
\hline 104 & Oncidium sarcodes Lindl. & $\mathrm{E}$ & $\mathrm{O}$ & $\mathrm{x}$ & & $\mathrm{x}$ & & $\mathrm{x}$ & $\mathrm{x}$ \\
\hline 105 & Oncidium varicosum Lindl. & $\mathrm{E}$ & $\mathrm{O}$ & $\mathrm{x}$ & $\mathrm{x}$ & $\mathrm{x}$ & $\mathrm{x}$ & $\mathrm{x}$ & \\
\hline 106 & Ornithophora radicans (Rchb.f.) Garay \& Pabst & $\mathrm{E}$ & $\mathrm{O}$ & $\mathrm{x}$ & $\mathrm{x}$ & $\mathrm{x}$ & $\mathrm{x}$ & & \\
\hline 107 & Phymatidium delicatulum Lindl. & $\mathrm{E}$ & $\mathrm{O}$ & $\mathrm{x}$ & $\mathrm{x}$ & $\mathrm{x}$ & $\mathrm{x}$ & $\mathrm{x}$ & $\mathrm{x}$ \\
\hline 108 & Phymatidium hysteranthum Barb.Rodr. & $\mathrm{E}$ & $\mathrm{R}$ & & & $\mathrm{x}$ & & & \\
\hline 109 & Platystele pygmaea (Hoehne) Pabst & $\mathrm{E}$ & $\mathrm{R}$ & & & & $\mathrm{x}$ & & \\
\hline 110 & Pleurothallis aff. saundersiana Rchb.f. & $\mathrm{E}$ & $\mathrm{R}$ & $\mathrm{x}$ & & & & & \\
\hline 111 & Pleurothallis arcuata Lindl. & $\mathrm{E}$ & $\mathrm{R}$ & & & & $\mathrm{x}$ & & \\
\hline 112 & Pleurothallis barbacenensis Barb.Rodr. & $\mathrm{E}$ & $\mathrm{R}$ & & & $\mathrm{x}$ & & & $\mathrm{x}$ \\
\hline 113 & Pleurothallis dryadum Schltr. & $\mathrm{E}$ & $\mathrm{R}$ & $\mathrm{x}$ & & & $\mathrm{x}$ & & \\
\hline 114 & Pleurothallis fusca Lindl. & $\mathrm{E}$ & $\mathrm{O}$ & $\mathrm{x}$ & $\mathrm{x}$ & $\mathrm{x}$ & $\mathrm{x}$ & & \\
\hline 115 & Pleurothallis grobyi Bateman ex Lindl. & $\mathrm{E}$ & $\mathrm{R}$ & & & $\mathrm{x}$ & & & \\
\hline 116 & Pleurothallis heterophylla (Barb.Rodr.) Cogn. & $\mathrm{E}$ & $\mathrm{R}$ & & & & $\mathrm{x}$ & & \\
\hline 117 & Pleurothallis hians Lindl. & $\mathrm{E}$ & $\mathrm{O}$ & $\mathrm{x}$ & $\mathrm{x}$ & $\mathrm{x}$ & $\mathrm{x}$ & & \\
\hline 118 & Pleurothallis klotzschiana Rchb.f. & $\mathrm{E}$ & & & & & & & $\mathrm{x}$ \\
\hline 119 & Pleurothallis leptotifolia Barb.Rodr. & $\mathrm{E}$ & $\mathrm{R}$ & $\mathrm{x}$ & & $\mathrm{x}$ & & & \\
\hline 120 & Pleurothallis linearifolia Cogn. & $\mathrm{E}$ & $\mathrm{R}$ & & & $\mathrm{x}$ & $\mathrm{x}$ & & \\
\hline 121 & Pleurothallis mouraeoides Hoehne & $\mathrm{E}$ & $\mathrm{R}$ & & & & $\mathrm{x}$ & & \\
\hline 122 & Pleurothallis murexoidea Pabst & $\mathrm{E}$ & $\mathrm{R}$ & & & $\mathrm{x}$ & & & \\
\hline 123 & Pleurothallis podoglossa Hoehne & $\mathrm{E}$ & $\mathrm{R}$ & $\mathrm{x}$ & & & $\mathrm{x}$ & & \\
\hline 124 & Pleurothallis pterophora Cogn. & $\mathrm{E}$ & $\mathrm{R}$ & $\mathrm{x}$ & & & $\mathrm{x}$ & & \\
\hline 125 & Pleurothallis quadridentata Barb.Rodr. & $\mathrm{E}$ & $\mathrm{R}$ & & & $\mathrm{x}$ & & & \\
\hline 126 & Pleurothallis rubens Lindl. & $\mathrm{E}$ & $\mathrm{O}$ & $\mathrm{x}$ & $\mathrm{x}$ & $\mathrm{x}$ & $\mathrm{x}$ & $\mathrm{x}$ & $\mathrm{x}$ \\
\hline 127 & Pleurothallis sarracenia Luer & $\mathrm{E}$ & $\mathrm{R}$ & & & $\mathrm{x}$ & & & \\
\hline 128 & Pleurothallis saundersiana Rchb.f. & $\mathrm{E}$ & $\mathrm{O}$ & $\mathrm{x}$ & $\mathrm{x}$ & $\mathrm{x}$ & $\mathrm{x}$ & $\mathrm{x}$ & \\
\hline 129 & Pleurothallis saurocephala Lodd. & $\mathrm{E}$ & $\mathrm{O}$ & $\mathrm{x}$ & $\mathrm{x}$ & $\mathrm{x}$ & $\mathrm{x}$ & $\mathrm{x}$ & \\
\hline 130 & Pleurothallis serpentula Barb.Rodr. & $\mathrm{E}$ & $\mathrm{R}$ & & & $\mathrm{x}$ & & & \\
\hline 131 & Pleurothallis sonderiana Rchb.f. & $\mathrm{E}$ & $\mathrm{R}$ & $\mathrm{x}$ & $\mathrm{x}$ & $\mathrm{x}$ & $\mathrm{x}$ & & \\
\hline 132 & Pleurothallis uniflora Lindl. & $\mathrm{E}$ & $\mathrm{R}$ & & & $\mathrm{x}$ & & & \\
\hline 133 & Pleurothallis warmingiii Rchb.f. & $\mathrm{E} / \mathrm{R}$ & $\mathrm{R}$ & & & $\mathrm{x}$ & & & \\
\hline 134 & Pleurothallis sp. 1 & $\mathrm{E}$ & $\mathrm{R}$ & & & & $\mathrm{x}$ & & \\
\hline 135 & Pleurothallis sp. 2 & $\mathrm{E}$ & $\mathrm{R}$ & & & $\mathrm{x}$ & $\mathrm{x}$ & & \\
\hline 136 & Polystachya estrellensis Rchb.f. & $\mathrm{E}$ & $\mathrm{O}$ & $\mathrm{x}$ & $\mathrm{x}$ & $\mathrm{x}$ & $\mathrm{x}$ & $\mathrm{x}$ & \\
\hline 137 & Prescottia oligantha (Sw.) Lindl & $\mathrm{T}$ & $\mathrm{R}$ & $\mathrm{x}$ & & $\mathrm{x}$ & $\mathrm{x}$ & $\mathrm{x}$ & \\
\hline 138 & Prescottia sp. & $\mathrm{T}$ & $\mathrm{O}$ & $\mathrm{x}$ & & $\mathrm{x}$ & & & \\
\hline 139 & Prescottia stachyodes (Sw.) Lindl. & $\mathrm{T}$ & $\mathrm{O}$ & $\mathrm{x}$ & $\mathrm{x}$ & $\mathrm{x}$ & $\mathrm{x}$ & $\mathrm{x}$ & \\
\hline 140 & Promenaea xanthina Lindl. & $\mathrm{E}$ & $\mathrm{R}$ & & & $\mathrm{x}$ & $\mathrm{x}$ & & \\
\hline
\end{tabular}

continua to be continued 
continuação - Tabela 1

continuation - Table 1

\begin{tabular}{|c|c|c|c|c|c|c|c|c|c|}
\hline $\mathrm{N}$ & Espécie & $\mathrm{Hb}$ & Oc & $\mathrm{C}$ & $\mathrm{E}$ & $\mathrm{Pg}$ & $\mathrm{P}$ & Ac & $\mathrm{Sl}$ \\
\hline 141 & Psilochilus modestus Barb.Rodr. & $\mathrm{E}$ & $\mathrm{R}$ & $\mathrm{x}$ & & & $\mathrm{x}$ & & \\
\hline 142 & Rodriguezia cf. obtusifolia (Lindl.) Rchb.f. & $\mathrm{E}$ & $\mathrm{R}$ & $\mathrm{x}$ & & $\mathrm{x}$ & & & \\
\hline 143 & Rodrigueziella handroi (Hoehne) Pabst & $\mathrm{E}$ & $\mathrm{R}$ & $\mathrm{x}$ & & $\mathrm{x}$ & & & \\
\hline 144 & Sarcoglottis sp. & $\mathrm{T}$ & $\mathrm{R}$ & & $\mathrm{x}$ & & & & $\mathrm{x}$ \\
\hline 145 & Sauroglossum nitidum (Vell.) Schltr. & $\mathrm{T}$ & $\mathrm{O}$ & $\mathrm{x}$ & $\mathrm{x}$ & $\mathrm{x}$ & $\mathrm{x}$ & $\mathrm{x}$ & \\
\hline 146 & Scaphyglottis reflexa Lindl. & $\mathrm{E}$ & $\mathrm{R}$ & & & $\mathrm{x}$ & & & \\
\hline 147 & Sophronitella violacea (Lindl.) Schltr & $\mathrm{E}$ & $\mathrm{R}$ & $\mathrm{x}$ & & & & & \\
\hline 148 & Stelis deregularis Barb.Rodr. & $\mathrm{E}$ & & & & & & & $\mathrm{x}$ \\
\hline 149 & Stelis modesta Barb.Rodr. & $\mathrm{E}$ & $\mathrm{R}$ & $\mathrm{x}$ & $\mathrm{x}$ & $\mathrm{x}$ & $\mathrm{x}$ & & \\
\hline 150 & Stelis peliochyla Barb.Rodr. & $\mathrm{E}$ & $\mathrm{R}$ & & & $\mathrm{x}$ & & & \\
\hline 151 & Stelis pterostele Hoehne \&e Schltr. & $\mathrm{E}$ & $\mathrm{O}$ & & $\mathrm{x}$ & $\mathrm{x}$ & $\mathrm{x}$ & & \\
\hline 152 & Stenorrhynchos lanceolatum (Aubl.)L.C.Rich. & $\mathrm{T}$ & $\mathrm{O}$ & $\mathrm{x}$ & $\mathrm{x}$ & $\mathrm{x}$ & $\mathrm{x}$ & $\mathrm{x}$ & \\
\hline 153 & Tetragamestus modestus Rchb.f. & $\mathrm{E}$ & $\mathrm{R}$ & & & & $\mathrm{x}$ & & \\
\hline 154 & Vanilla chamissonis Klotzsch & $\mathrm{E}$ & $\mathrm{R}$ & & $\mathrm{x}$ & $\mathrm{x}$ & & & \\
\hline 155 & Vanilla edwallii Hoehne & $\mathrm{E}$ & $\mathrm{O}$ & $\mathrm{x}$ & $\mathrm{x}$ & $\mathrm{x}$ & $\mathrm{x}$ & $\mathrm{x}$ & $\mathrm{x}$ \\
\hline 156 & Warrea warreana (Lodd. ex Lindl.) C.Schweinf. & $\mathrm{T}$ & $\mathrm{R}$ & & & $\mathrm{x}$ & $\mathrm{x}$ & $\mathrm{x}$ & \\
\hline 157 & Zygopetalum crinitum Lodd. & $\mathrm{E}$ & $\mathrm{R}$ & & & $\mathrm{x}$ & & & \\
\hline 158 & Zygopetalum mackayi Hook. & $\mathrm{R}$ & $\mathrm{R}$ & & $\mathrm{x}$ & & & & \\
\hline 159 & Zygopetalum maxillare Lodd. & E & $\mathrm{R}$ & $\mathrm{x}$ & $\mathrm{x}$ & $\mathrm{x}$ & $\mathrm{x}$ & & \\
\hline
\end{tabular}

Das coletas informadas pela rede SpeciesLink (SpeciesLink, 2014), algumas delas históricas para a Cantareira, podemos destacar as de Loefgren 1895, 1896, 1897; Edwall 1896, Usteri 1897; Hoehne 1918, 1919, 1932, entre outras, como os primeiros registros da família Orchidaceae para a região.

A documentação fotográfica resultou em fotos de 145 espécies floridas, que estão disponíveis como guia de campo publicado no Field Museum, disponível em: <http://fieldguides.fieldmuseum.org/ guides/guide/444>.

Em comparação com outros levantamentos feitos em São Paulo e regiões próximas, o Parque Estadual da Cantareira, com 159 espécies, revela um número superior às demais áreas estudadas, como no Parque Estadual da Ilha do Cardoso (147 espécies, Romanini e Barros, 2008), em Mogi das Cruzes, no Parque Municipal Chiquinho Veríssimo (70 espécies, Rodrigues e Barros, 2012) e ainda acima do encontrado na Serra do Japi em Jundiaí (125 espécies, Pansarin e Pansarin, 2008) ou no Parque Estadual das Fontes do Ipiranga - PEFI (125 espécies, Barros, 1983).
Esse número também foi superior ao encontrado por Breier (2005) no Parque Estadual Carlos Botelho (42 espécies) ou Catharino e Barros (2004) no Maciço da Jureia (Estação Ecológica Jureia-Itatins, 77 espécies). Assim, foi a área mais rica entre as estudadas até o momento em São Paulo. A alta riqueza de Orchidaceae para o PEC é fator importante para justificar esforços para a sua conservação e ampliação de levantamentos biológicos nesta unidade de conservação paulista.

Pleurothallis foi o gênero melhor representado, com 26 espécies, seguido por Oncidium com 14 espécies, Cyclopogon com oito espécies, Habenaria e Octomeria com sete espécies, Epidendrum e Maxillaria, com seis espécies, Capanemia, Campylocentrum e Barbosella com quatro espécies, Eurystyles, Erytrodes, Stelis, Prescotia e Zygopetalum com três espécies, Bifrenaria, Catasetum, Dichaea, Encyclia, Gomesa, Isabelia, Phymatidium, Malaxis, Bulbophyllum e Vanilla com duas espécies. Os outros 36 gêneros foram representados por uma espécie. 
Considerando a abundância das espécies, verificou-se que oito espécies foram classificadas como abundantes, 46 foram consideradas ocasionais, e 98, raras. Levando-se em consideração o grau de ameaça, verificou-se que dez espécies estão presentes em listas vermelhas de flora ameaçada de extinção (São Paulo, 2004; Brasil, 2008; Martinelli e Moraes, 2013) em categorias variadas (Tabela 2).
As seis espécies referidas como presumivelmente extintas (EX) (Tabela 2) foram também classificadas como raras (Tabela 1), pois, apesar de presentes, o número reduzido de indivíduos avistados e a ausência de frutos sugerem populações em declínio, necessitando, ainda, de atenção especial quanto à conservação. Dessa maneira, ficariam melhor posicionadas em categoria de alto risco, devendo sua classificação ser alterada para "em perigo crítico" (CR).

Tabela 2. Orquídeas constantes em listas de flora ameaçada de extinção válidas para São Paulo (São Paulo, 2004; Brasil, 2008; Martinelli e Moraes, 2013), registradas nos diferentes núcleos/trilhas do PEC, categorias de ameaça - Cat: em perigo crítico - $\mathrm{CR}$, vulnerável - VU, presumivelmente extinta - EX.

Table 2. Orchids present in lists of flora threatened with extinction valid for São Paulo (São Paulo, 2004; Brasil, 2008; Martinelli and Moraes, 2013), recorded in different nuclei/tracks in PEC, threat categories - Cat: critically endangered - CR, vulnerable - VU, presumably extinct - EX.

\begin{tabular}{llll}
\hline & Espécie & Cat & Listas \\
\hline 1 & Centroglossa macroceras Barb.Rodr. & EX & São Paulo (2004) \\
2 & Isabelia virginalis Barb.Rodr. & EX & São Paulo (2004); Martinelli e Moraes (2013) \\
3 & Laelia virens Lindl. & EX & Brasil (2008) \\
4 & Lankesterella epiphyta (Barb.Rodr.) Mansf. & EX & São Paulo (2004) \\
5 & Lycaste rossyi Hoehne & CR & São Paulo (2004) \\
6 & Oncidium divaricatum (Lindl.) Beer & EX & São Paulo (2004); Martinelli e Moraes (2013) \\
7 & Oncidium hians Lindl. & EX & São Paulo (2004); Martinelli e Moraes (2013) \\
8 & Oncidium praetextum Rchb.f. & VU & São Paulo (2004) \\
9 & Oncidium pubes Lindl. & VU & São Paulo (2004) \\
10 & Zygopetalum maxillare Lodd. & VU & São Paulo (2004) \\
\hline
\end{tabular}

Por outro lado, outras 88 espécies não presentes em listas vermelhas foram classificadas como raras, sendo localmente ameaçadas, da mesma forma como as constantes em listas, por terem sido avistadas em número menor ou igual a cinco indivíduos, em áreas de floresta madura e não terem sido observados frutos nem plântulas nas proximidades. Isso implica em maior atenção para a conservação de tais espécies, podendo todas ser consideradas minimamente vulneráveis, mesmo porque podem ser alvo de coletas extrativistas e/ou extinção local por serem populações em declínio natural no PEC.
Primack e Rodrigues (2001) elencam diversas categorias de vulnerabilidade à extinção, entre elas, espécies que requerem nichos específicos, espécies com área de ocorrência limitada, espécies com uma ou algumas populações, espécies com populações pequenas, espécies que são características de ambientes estáveis, espécies que sofrem extrativismo, uma vez que estas características de suscetibilidade à extinção ocorrem em conjunto e indicam a necessidade de ações integradas de conservação da família Orchidaceae no Parque Estadual da Cantareira. 
Grande parte das espécies raras e outras dez citadas em listas vermelhas (São Paulo, 2004; Brasil, 2008; Martineli e Moraes, 2013), registradas para o PEC, ocorrem em áreas restritas e em número reduzido de indivíduos, muitas delas não apresentando frutos nem propágulos próximos, sugerindo populações em declínio e a necessidade de atenção especial à sua conservação. Dessa maneira, sugere-se sua permanência em categoria de alto risco de extinção.

Algumas espécies só foram registradas na floresta madura e em seus remanescentes e podem ser consideradas indicadoras desta fitofisionomia, como: Amblostoma armeniacum (Lindl.) Brieger, Amblostoma tridactylum (Lindl.) Rchb.f., Barbosella australis (Cogn.) Schltr., Barbosella dusenii (A.Samp.) Schltr., Barbosella microphylla (Barb.Rodr.) Schltr., Barbosella miersii (Lindl.) Schltr., Bifrenaria aureofulva Lindl., Bifrenaria harrisoniae (Hook.) Rchb.f., Bulbophyllum napellii Lindl., Capanemia superflua (Rchb.f.) Garay, Centroglossa macroceras Barb.Rodr., Dichaea pendula (Aubl.) Cogn., Epidendrum proligerum Barb.Rodr., Erythrodes lacteola (Barb.Rodr.) Ames, Erythrodes picta (Lindl.) Ames, Isabelia virginalis Barb.Rodr., Laelia virens Lindl., Lankesterella epiphyta (Barb.Rodr.) Mansf., Lycaste rossyi Hoehne, Malaxis excavata (Lindl.) Kuntze, Malaxis parthoni C.Morren, Maxillaria cerifera Barb.Rodr., Maxillaria cogniauxiana Hoehne, Maxillaria gracilis Lodd., Maxillaria madida Lindl., Maxillaria marginata (Lindl.) Fenzl, Maxillaria picta Hook., Octomeria concolor Barb.Rodr., Octomeria crassifolia Lindl., Octomeria diaphana Lindl., Octomeria grandiflora Lindl., Octomeria juncifolia Barb.Rodr., Oncidium crispum Lodd., Oncidium divaricatum (Lindl.) Beer, Oncidium forbesii Hook., Oncidium gardneri Lindl., Oncidium harrisonianum Lindl., Oncidium hians Lindl., Oncidium longipes Lindl., Oncidium praetextum Rchb.f., Ornithophora radicans (Rchb.f.) Garay \& Pabst, Platystele pygmaea (Hoehne) Pabst, Pleurothallis aff. saundersiana Rchb.f., Pleurothallis arcuata Lindl., Pleurothallis dryadum Schltr., Pleurothallis fusca Lindl., Pleurothallis grobyi Bateman ex Lindl., Pleurothallis heterophylla (Barb.Rodr.) Cogn., Pleurothallis hians Lindl., Pleurothallis klotzschiana Rchb.f., Pleurothallis leptotifolia Barb. Rodr.,
Pleurothallis linearifolia Cogn., Pleurothallis mouraeoides Hoehne, Pleurothallis murexoidea Pabst, Pleurothallis podoglossa Hoehne, Pleurothallis pterophora Cogn., Pleurothallis quadridentata Barb. Rodr., Pleurothallis sarracenia Luer, Pleurothallis saundersiana Rchb.f., Pleurothallis serpentula Barb.Rodr., Pleurothallis uniflora Lindl., Promenaea xanthina Lindl., Psilochilus modestus Barb.Rodr., Rodrigueziella handroi (Hoehne) Pabst, Scaphyglottis reflexa Lindl., Sophronitella violacea (Lindl.) Schltr., Stelis modesta Barb.Rodr., Stelis peliochyla Barb.Rodr., Stelis pterostele Hoehne \& Schltr., Zygopetalum crinitum Lodd., Zygopetalum maxillare Lodd.

\subsection{Similaridade Interna Entre Áreas do PEC}

A análise de agrupamento da distribuição das espécies por núcleo/trilha, ilustrada na Figura 3, evidencia a separação de Pedra Grande - PG) por se mostrar a área mais rica com 114 espécies. Apesar de ter poucos trechos de floresta madura, tem amplas áreas de florestas secundárias contendo pequenas manchas de florestas maduras em seu interior, congregando, portanto, tanto espécies características de florestas menos úmidas e secundárias quanto espécies características de florestas mais úmidas e maduras. Também o Núcleo Águas Claras com 52 espécies se separa dos demais por apresentar a menor riqueza, pois nele predominam florestas secundárias em regeneração sob plantios de espécies exóticas. Por outro lado, evidencia-se o grupo formado por Pinheirinho - Pin com 97 espécies, Engordador - Eng com 82, e Cabuçú - Cab com 86. Engordador e Cabuçú possuem trechos de floresta madura e secundária em regeneração. Pinheirinho, que apresentou a maior riqueza em menor área amostral, possui os trechos mais significativos de floresta madura, consequentemente, mais espécies de orquídeas típicas destas florestas estão presentes.

Deve-se ressaltar que os núcleos/trilhas apresentam áreas variadas, diferentes fitofisionomias associadas à variação de relevo, altitude, umidade e histórico de conservação. Verifica-se que a riqueza de espécies tende a aumentar nos fragmentos mais preservados e nas áreas próximas a cursos d'água. 

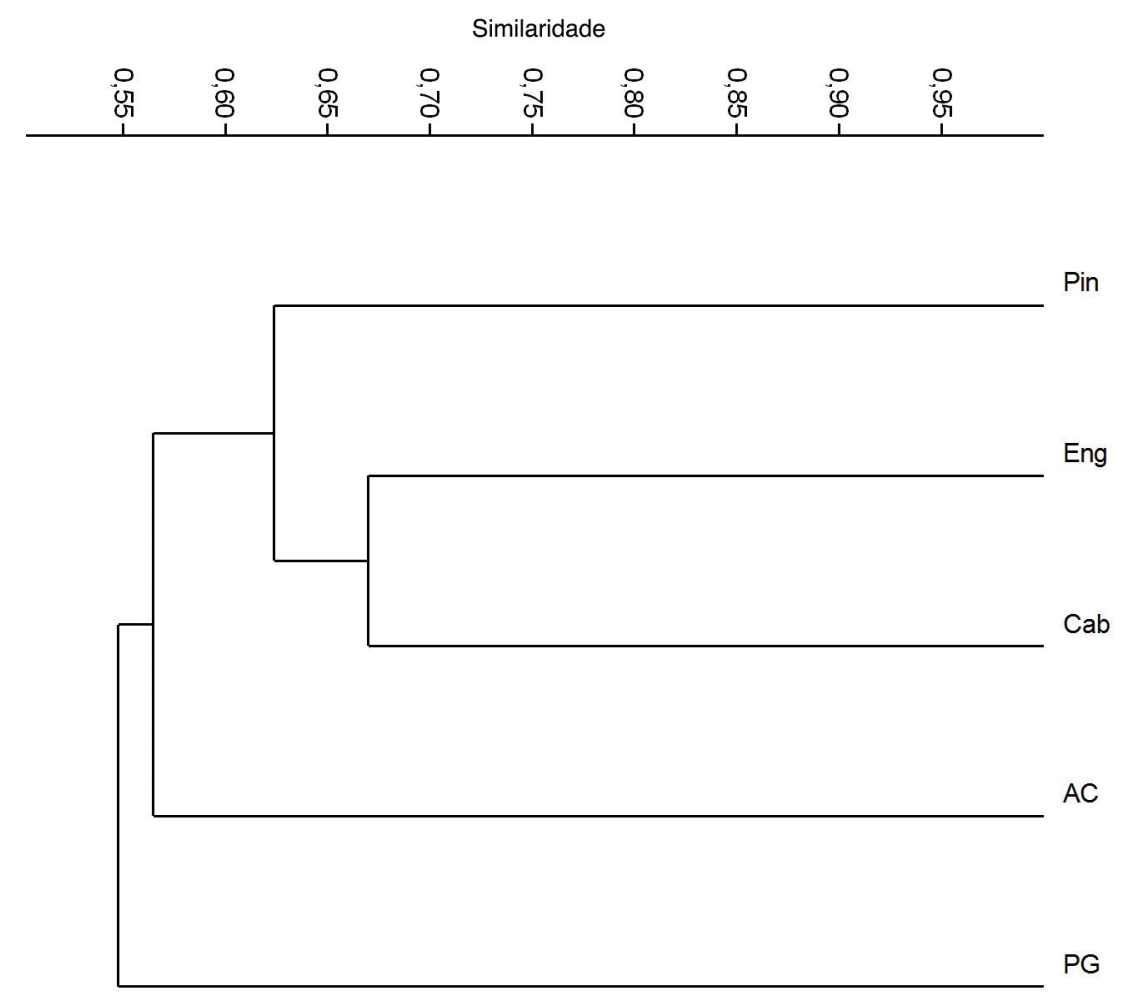

Figura 3. Agrupamento por UPGMA, utilizando o índice de Jaccard, efetuado a partir da presença/ausência de 150 espécies de Orchidaceae nos cinco núcleos do PEC (não considerando espécies adicionadas pela consulta ao SpeciesLink): Pedra Grande - PG, Engordador - Eng, Águas Claras - AC, Cabuçú - Cab e Pinheirinho - Pin (coeficiente de correlação cofenética $=0,8017$ ).

Figure 3. Grouping UPGMA using Jaccard index made from the occurrence of 150 species of Orchidaceae in the five nuclei of PEC (species added after consulting the SpeciesLink were not considered): Pedra Grande - PG, Engordador - Eng, Águas Claras $-\mathrm{AC}, \mathrm{Cabuçu}-\mathrm{Cab}$ and Pinheirinho - Pin (cophenetic correlation coefficient $=0.8017$ ). 


\subsection{Conservação in situ: Realocação de Plantas na Natureza}

As orquídeas encontradas em árvores e galhos caídos foram resgatadas, dando-se preferência à remoção da planta com parte do substrato visando minimizar os danos sobre o sistema radicular, garantindo melhores condições de sobrevivência aos exemplares, que após tratamento fitossanitário, foram realocadas no habitat nas condições ideais para cada espécie.

Algumas plantas de cada espécie foram realocadas no entorno do Centro de Visitantes e nas trilhas de visitação e são utilizadas em atividades de educação para a conservação. Foram resgatadas 3.120 orquídeas de 105 espécies, das quais 450 foram incluídas em coleções científicas do IBt e PEC e 2.670 realocadas na natureza.

\subsection{Conservação ex situ: Inclusão de Plantas em Coleções}

Parte das plantas provenientes de resgate em árvores e galhos caídos, foi incluída nas coleções do Orquidário Frederico Carlos Hoehne - IBt e do Parque Estadual da Cantareira, fornecendo material para documentação em herbário, fotografias e identificação posterior, além de servir como instrumento de educação para a conservação.

O cultivo das plantas em estufa, no Núcleo Pedra Grande, facilitou os estudos e aumentou a chance de sobrevivência das plantas resgatadas por permanecerem no próprio habitat. $\mathrm{O}$ trabalho de resgate, por sua vez, vem se mostrando muito útil ao conhecimento, identificação botânica e conservação da família Orchidaceae no Parque Estadual da Cantareira.

\subsection{Atividades de Educação para a Conservação}

Foi elaborado um minicurso intitulado Reconhecimento e Identificação de Orquídeas em Trilhas, sendo ministrado aos educadores ambientais de todos os núcleos. Durante o minicurso, ministrado semestralmeste, os educadores foram apresentados à família Orchidaceae e às principais espécies presentes na coleção viva e trilhas de visitação, além de participarem de atividades de capacitação para identificação, cultivo, resgate das orquídeas em árvores e galhos caídos e sua posterior realocação. A coleção viva, montada com as espécies resgatadas no Núcleo Pedra Grande, foi adequada para ser utilizada durante a visitação, realizada diariamente. Foram elaboradas placas interpretativas, um manual ilustrado impresso na forma de banner e observadores feitos de bambu, adaptados de Honig (2000), para facilitar a visualização das orquídeas realocadas nas árvores no entorno da coleção.

Foram realizadas oficinas mensais com o objetivo de sensibilizar os visitantes sobre a necessidade de preservação dos recursos naturais, divulgando boas práticas ambientais no manejo, cultivo e realocação. Ao final do roteiro, cada turma teve a opção de realocar uma orquídea resgatada nas árvores do Centro de Visitantes.

O manual ilustrado (Figura 4) elaborado, foi adaptado e impresso no formato banner $(1,2 \mathrm{~m} \times 2 \mathrm{~m})$ e afixado junto à coleção viva no PEC para ser usado complementando as visitas escolares, contém características da família Orchidaceae e fotos de algumas espécies mais notáveis que podem ser observadas na coleção e Centro de Visitantes.

A coleção viva e as árvores do entorno que receberam plantas realocadas foram utilizadas nas atividades de educação para a conservação e observou-se grande interesse dos visitantes pela coleção do orquidário e também pela utilização dos observadores de bambu na visualização das plantas realocadas. 


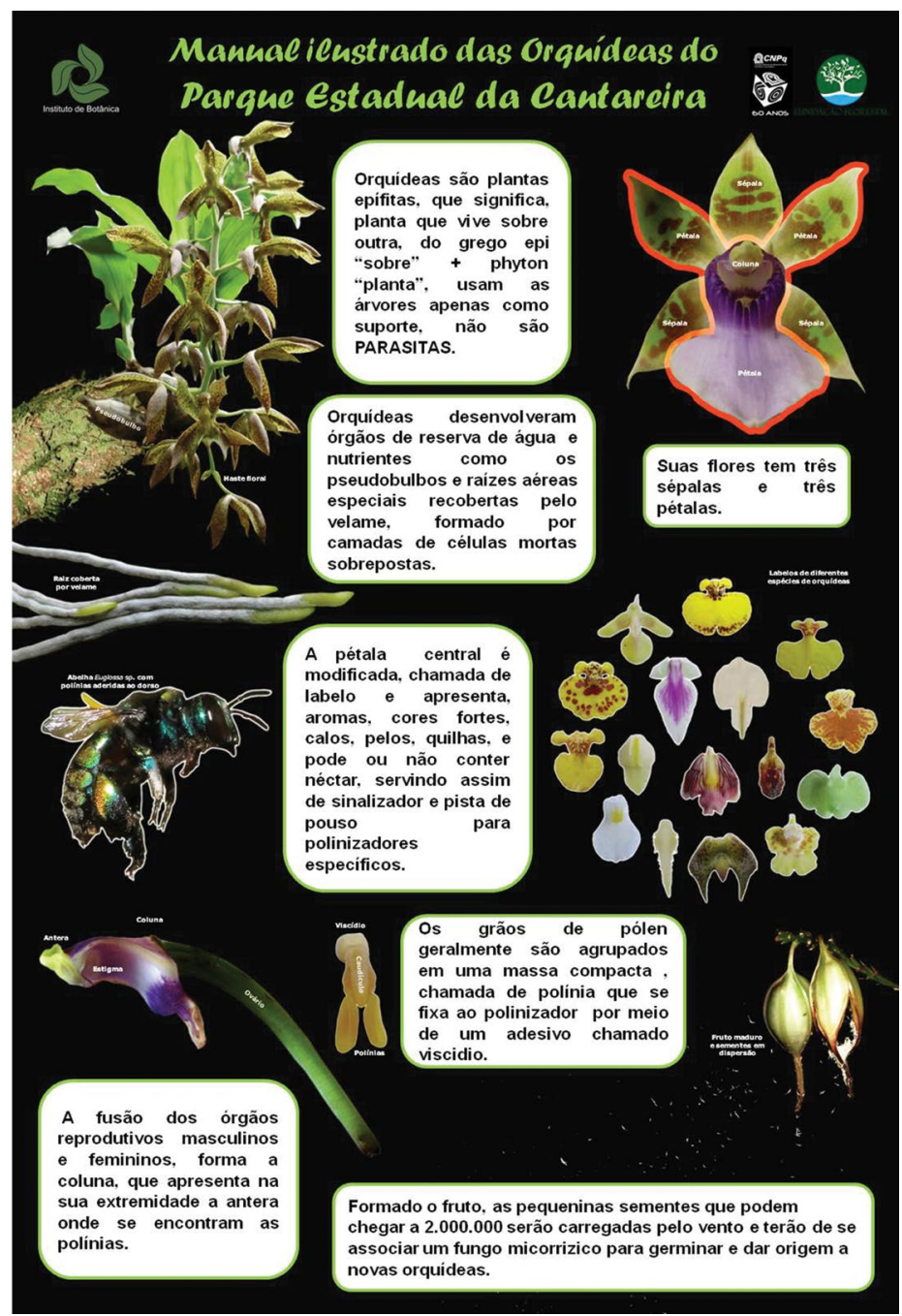

Figura 4. Banner elaborado para a estufa do PEC, adaptado a partir do manual ilustrado, para uso em atividades educativas.

Figure 4. Banner prepared for the PEC greenhouse, adapted from the illustrated handbook for use in educational activities.

\section{CONCLUSÕES}

Tendo em vista a listagem de 159 espécies, maior número de orquídeas já registrado nos estudos em áreas específicas em São Paulo, pode-se concluir que o Parque Estadual da Cantareira é a unidade de conservação paulista com maior riqueza da família Orchidaceae registrada até o momento, o que torna necessária a concentração de esforços a fim de conservá-la. Concluimos também que, entre essas espécies, algumas podem ser consideradas indicadoras da floresta madura e de seus remanescentes.

Sugere-se a ampliação dos estudos incluindo ações conjuntas como conservação in situ por meio do resgate e realocação e implementação da conservação ex situ por meio da inclusão de plantas coletadas em coleções científicas, possibilitando a micropropagação das espécies raras e ameaçadas, fornecendo propágulos para a reintrodução nas áreas do PEC e oferecendo plantas nativas propagadas para o mercado orquidófilo para diminuir a coleta e o comércio ilegais de plantas nativas retiradas das matas, além da implementação de atividades educativas considerando a família como foco. A metodologia de resgate e cultivo, além do grande período de coletas, proporcionou uma listagem ampla de espécies, uma vez que as plantas foram acompanhadas até a floração e posterior identificação, o que seria mais difícil de realizar somente com observação de exemplares floridos no habitat. 
O desenvolvimento de atividades educativas, proporcionando a transposição de dados científicos em atividades e material didático, e a elaboração do manual ilustrado e placas interpretativas para a coleção viva, associada ao uso em atividades de educação para a conservação, mostrou que as orquídeas despertam a curiosidade, sendo possível abordar inúmeros temas como: biologia floral, polinização, morfologia vegetal, além abordar inúmeros assuntos relacionados à problemática ambiental atual e à conservação dos recursos naturais renováveis.

Tendo em vista a observação da visitação realizada na coleção viva montada no PEC, as primeiras impressões nos levam a crer que Orchidaceae, se utilizada como uma família-bandeira, pode ser uma excelente ferramenta para as atividades de educação para a conservação ali realizadas.

\section{AGRADECIMENTOS}

O autor Luciano Ramos Zandoná agradece aos amigos Servilio Mendes, Lucas Borges e Eitor Bastos, pela ajuda indispensável em campo; ao $\mathrm{CNPq}$; ao Instituto de Botânica de São Paulo, por toda estrutura; aos membros da banca, Fábio de Barros e Frederico Arzolla; aos gestores do Parque Estadual da Cantareira, Fernando Descio e Vladimir Arrais, pela recepção amiga e por toda infraestrutura; aos funcionários, educadores ambientais e amigos do Parque Estadual da Cantareira, pela ajuda indispensável ao estudo; à Fundação Florestal e ao Instituto Florestal, pelas autorizações e permissões para realização da pesquisa no Parque Estadual da Cantareira; aos funcionários do Herbário Dom Bento Pickel - SPSF; e aos revisores anônimos, pela colaboração no manuscrito.

\section{REFERÊNCIAS BIBLIOGRÁFICAS}

ARZOLLA, F.A.R.D.P. Florestas secundárias e a regeneração natural de clareiras antrópicas, na Serra da Cantareira-SP. 2011. 141 f. Tese (Doutorado em Biologia Vegetal) - Instituto de Biologia, UNICAMP, Campinas.

BARROS, F. Flora fanerogâmica da Reserva do Parque Estadual das Fontes do Ipiranga: Orchidaceae. Hoehnea, v. 10, p. 74-124, 1983.
BARROS, F. et al. Orchidaceae. In: LISTA de espécies da flora do Brasil. Jardim Botânico do Rio de Janeiro. Disponível em: <http://floradobrasil. jbrj.gov.br/jabot/floradobrasil/FB179>. Acesso em: 12 mar. 2014.

BRASIL. Ministério do Meio Ambiente - MMA. Instrução Normativa $\mathrm{n}^{\circ} 6$, de 23 de setembro de 2008. Diponível em: <http://www.mma.gov.br/ estruturas/179/_arquivos/179_05122008033615.pdf $>$. Acesso em: 5 fev. 2014.

BREIER, T.B. O epifitismo vascular em florestas do sudeste do Brasil. 2005. 139 f. Tese (Doutorado em Biologia Vegetal) - Instituto de Biologia, Universidade Estadual de Campinas, Campinas.

CATHARINO, E.L.M; BARROS, F. Orquídeas do Maciço da Juréia e arredores. In: MARQUES, O.A.V.; DULEBA, W. (Ed.). Estação Ecológica Juréia-Itatins: ambiente físico, flora e fauna. Ribeirão Preto: Holos, 2004. p. 152-161.

DANTAS, A.G.M. Geologia da Faixa São Roque e intrusivas associadas na região entre São Paulo e Mairiporã, norte da Grande São Paulo. 1990. 199 f. Dissertação (Mestrado em Geoquímica e Geotectônica) - Instituto de Geociências, Universidade de São Paulo, São Paulo.

DISLICH, R.; MANTOVANI, W. A flora das epífitas vasculares da Reserva da Cidade Universitária "Armando Salles de Oliveira" (São Paulo, Brasil). Boletim de Botânica da Universidade de São Paulo, v. 17, p. 61-83, 1998.

DIXON, K.; PHILLIPS, R.D. The orchid conservation challenge. Lankesteriana, v. 1-2, p. 11-12, 2007.

DRESSLER, R.L. Phylogeny and classification of the orchid family. Portland: Dioscorides Press, 1993. 341 p.

. How many orchid species? Selbyana, v. 26, p. 155-158, 2005.

FILGUEIRAS, T.S. et al. Caminhamento - um método expedito para levantamentos florísticos qualitativos. Cadernos de Geociências, v. 2, n. 4, p. 39-43, 1994.

HAMMER, Ø.; HARPER, D.A.T.; RYAN, P.D. PAST: Paleontological statistics software package for education and data analysis. Palaeontologia Electronica, v. 4, n. 1, 9 p., 2001. Disponível em: $<$ http://palaeo-electronica.org/2001_1/past/ issue1_01.htm>. Acesso em: 5 fev. 2014. 
HIETZ, P. Diversity and conservation of epiphytes in a changing environment. In: THE INTERNATIONAL CONFERENCE ON BIODIVERSITY AND BIORESOURCES: CONSERVATION AND UTILIZATION, IUPAC, 1999, Phuket, Thailand. Disponível em: http:// www.iupac.org/symposia/proceedings/phuket97/ hietz.pdf. Acesso em: 16 fev. 2014.

HOEHNE, F.C. Iconografia de Orchidaceas do Brasil. São Paulo: Secretaria da Agricultura, 1949. $614 \mathrm{p}$.

HONIG, M. Como dar vida ao seu jardim! Interpretação ambiental em jardins botânicos. Tradução Maria Teresa Bernardes. Rio de Janeiro: RBJB, 2000. 92 p.

MARTINELLI, G.; MORAES, M.A. Livro vermelho da flora do Brasil. Rio de Janeiro: Andrea Jakobsson: Instituto de Pesquisas Jardim Botânico do Rio de Janeiro, 2013. 1100 p.

MIRENDA, T.J. Botanic gardens, education, andorchid conservation strategies: the need for a coordinated approach. Lankesteriana, v. 11, n. 3, p. 301-305, 2011.

MYERS, N. et. al. Biodiversity hotspots for conservation priorities. Nature, v. 403, p. 853-858, 2000.

PABST, G.F; DUNGS, F. Orchidaceae brasiliensis. Hildeshein: Brücke-Verlag Kurt Schmersow, 1975. v. 1.

Schmersow, 1977. v. 2.

Hildeshein: Brücke-Verlag Kurt

PANSARIN, E.R.; PANSARIN, L.M. A família Orchidaceae na Serra do Japi, São Paulo, Brasil. Rodriguésia, v. 59, n. 1, p. 99-111, 2008.

PILIACKAS, J.M.; BARBOSA, L.M.; CATHARINO, E.L.M. Levantamento das epífitas vasculares do manguezal do Rio Picinguaba, Ubatuba, São Paulo. In: WATANABE, S. (Coord.). SIMPÓSIO DE ECOSSISTEMAS BRASILEIROS, 5., 2000, São Paulo. Anais... São Paulo: Aciesp, 2000. v. 2, p. 357-363.

PRIMACK, R.B.; RODRIGUES, E. Biologia da conservação. Londrina: Planta, 2001. 328 p.
RASSMUSSEN, H.N.; RASSMUSSEN, F.N. The role of botanical gardens in orchid research. In: HEYWOOD, V.H.; JACKSON, P.S. (Ed.). Tropical botanic gardens. Cambridge: Cambridge University Press, 1991. p. 275-287.

RODRIGUES, V.T.; BARROS, F. A família Orchidaceae na Serra do Itapeti. In: MORINI, M.S. de C.; MIRANDA, V.F.O. de. (Org.). Serra do Itapeti: aspectos históricos, sociais e naturalísticos. Bauru: Canal 6, 2012. v. 1, p. 1-400.

ROMANINI, R.P.; BARROS, F. Orchidaceae. In: MELO, M.M.R.F. et al. (Ed.). Flora fanerogâmica da Ilha do Cardoso. São Paulo: Instituto de Botânica, 2008. v. 12, p. 29-275.

SANTOS, A.C.L. Composição florística e estrutura da comunidade de epífitas vasculares associadas a trilhas no Parque Estadual das Fontes do Ipiranga, São Paulo, SP, Brasil. 2008. 56 f. Dissertação (Mestrado em Biologia Vegetal) Instituto de Botânica, São Paulo.

SÃO PAULO (Estado). Secretaria do Meio Ambiente. Resolução SMA 48, de 21 de setembro de 2004. Espécies da flora ameaçada de extinção do Estado de São Paulo. Diário Oficial do Estado São Paulo, Poder Executivo, v. 114, n. 179, 22 set. 2004. Seção I, p. 26.

Secretaria do Meio Ambiente. Plano de Manejo do Parque Estadual da Cantareira. São Paulo: Fundação Florestal, 2010. 586 p.

SEATON, T.P. Orchid conservation: where do we go from here? Lankesteriana, v. 7, n. 1-2, p. 13-16, 2007.

SPECIESLINK. Disponível em: <http://www. splink.org.br>. Acesso em: 30 set. 2014 .

STEWART, J. Orchids in botanic gardens - necessity or luxury? In: HEYWOOD, V.H.; JACKSON, P.S. (Ed.). Tropical botanic gardens. Cambridge: Cambridge University Press, 1991. p. 267-273.

ZANDONÁ, L.R. Orchidaceae no Parque Estadual da Cantareira: florística e conservação. 2014. 120 f. Dissertação (Mestrado em Biodiversidade Vegetal e Meio Ambiente) - Instituto de Botânica de São Paulo, São Paulo. 